\title{
Prevalence and Association of Congenital Anomalies According to the Maternal Body Mass Index: Cross-Sectional Study
}

\section{Prevalência e associação de anomalias congênitas de acordo com índice de massa corporal materno: Estudo transversal}

\author{
Carolina Leão de Moraes ${ }^{1}$ Carolina Rodrigues Mendonça ${ }^{1} \quad$ Natália Cruz e Melo ${ }^{2}$ \\ Waldemar Naves do Amaral ${ }^{1}$ \\ 1 Faculty of Medicine, Department of Obstetrics and Gynecology, Hospital \\ das Clínicas, Universidade Federal de Goiás, Goiânia, GO, Brazil \\ Address for correspondence Carolina Leão de Moraes, Msc, Av. \\ 2 Paulista Medical School, Universidade Federal de São Paulo, São \\ Paulo, SP, Brazil \\ Esperança, s/n, 74690-900 Chácaras de Recreio Samambaia, Goiânia, \\ GO, Brazil (e-mail: carolina.leao.moraes2@gmail.com).
}

Rev Bras Ginecol Obstet 2019;41:280-290.

\section{Abstract \\ Keywords \\ - congenital anomalies \\ - pregnancy \\ - obesity \\ - body weight \\ - fetal ultrasonography}

Objective To evaluate and compare the prevalence of structural congenital anomalies (CAs) according to maternal body mass index (BMI).

Methods The present cross-sectional study involved pregnant women with fetuses diagnosed with structural CAs through morphological ultrasonography between November 2014 and January 2016. The nutritional status of the pregnant women was classified according to the gross value of the body mass index. The pregnant women were categorized into four groups: low weight, adequate weight, overweight, and obesity. Statistical analysis was performed using Stata/SE version 12.0 (Stata Corporation, College Station, TX), with values of $p \leq 0.05$ considered statistically significant.

Results A total of 223 pregnant women had fetuses diagnosed with CAs. The prevalence of structural CAs in pregnant women with low weight was of $20.18 \%$, of $43.50 \%$ in pregnant women with adequate weight, of $22.87 \%$ in pregnant women with overweight, and of $13.45 \%$ in pregnant women with obesity. The prevalence of central nervous system (CNS) anomalies and of genitourinary system anomalies was high for the four groups of pregnant women. A positive association was observed between multiple anomalies in pregnant women with adequate weight (prevalence ratio $[\mathrm{PR}]=1.65 ; p \leq 0.004$ ) and between anomalies of the lymphatic system in obese pregnant women $(P R=4.04, p \leq 0.000)$. Conclusion The prevalence of CNS and genitourinary system anomalies was high in all of the BMI categories. Obese pregnancies were associated with lymphatic system anomalies. Therefore, screening and identification of the risk factors for CAs are important, regardless of the maternal BMI. Our findings reinforce the importance of discussing with pregnant women maternal nutrition and its effect on fetal development and on neonatal outcome.

(1DCarolina Leão de Moraes's ORCID is https://orcid.org/0000-00030755-0753.

received September 17, 2018 accepted

February 8, 2019
DOI https://doi.org/ $10.1055 / \mathrm{s}-0039-1683971$. ISSN $0100-7203$.
Copyright $\odot 2019$ by Thieme Revinter Publicações Ltda, Rio de Janeiro, Brazil
License terms

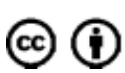




\section{Resumo}

\section{Palavras-chave}

- anomalias congênitas

- gravidez

- obesidade

- peso corporal

- ultrassonografia fetal
Objetivo Avaliar e comparar a prevalência de anomalias congênitas (ACs) estruturais de acordo com o índice de massa corporal (IMC) materno.

Métodos Estudo transversal envolvendo gestantes com fetos diagnosticados com ACs estruturais por ultrassonografia morfológica entre novembro de 2014 e janeiro de 2016. O estado nutricional das gestantes foi classificado de acordo com o valor bruto do índice de massa corporal. As gestantes foram categorizadas em quatro grupos: baixo peso, peso adequado, sobrepeso e obesidade. A análise estatística foi realizada no programa Stata/SE versão 12.0 (Stata Corporation, College Station, TX), com valores de $p \leq 0,05$ considerados estatisticamente significantes.

Resultados Um total de 223 gestantes tiveram fetos diagnosticados com ACs. A prevalência de AC estrutural em gestantes com baixo peso foi de $20,18 \%$, em gestantes com peso adequado foi de $43,50 \%$, em gestantes com sobrepeso foi de $22,87 \%$, e em gestantes com obesidade foi de $13,45 \%$. A prevalência de anomalias do sistema nervoso central (SNC) e do sistema geniturinário foi alta para os quatro grupos. Observou-se associação positiva entre múltiplas anomalias em gestantes com peso adequado (razão de prevalência $[R P]=1,65 ; p \leq 0,004$ ) e entre anomalias do sistema linfático em gestantes obesas ( $R P=4,04, p \leq 0,000)$.

Conclusão A prevalência das anomalias do SNC e do sistema geniturinário foi alta em todas as categorias de IMC. Gestantes obesas foram associadas a anomalias do sistema linfático. Portanto, o rastreamento e a identificação dos fatores de risco para as AC são importantes, independentemente do IMC materno. Nossos achados reforçam a importância de discutir com gestantes sobre a nutrição materna e seu efeito no desenvolvimento fetal e no desfecho neonatal.

\section{Introduction}

Nutritional status and adequate maternal weight gain are important aspects for the health and the well-being before, during, and after pregnancy. ${ }^{1,2}$ It is known that women with normal gestational weight gain have fewer complications during pregnancy. ${ }^{3}$

In contrast, low birthweight and malnutrition in pregnant women have been related to adverse effects during pregnancy, such as spontaneous abortion, hypertensive disorders, and fetal growth restriction and prematurity., ${ }^{4,5}$ Maternal weight gain beyond the recommended limit may lead to complications in the pre- and postpartum period, and may represent an obstetric risk factor, with consequences for the mother and for the fetus. ${ }^{3,6}$ Risks to pregnant women include gestational diabetes mellitus (DM) and preeclampsia, whereas risks to fetuses include congenital anomalies (CAs), macrosomia, stillbirth, neonatal death, and prematurity. 3,7-9

Congenital anomalies result in mortality in approximately 276,000 newborns per year worldwide. Currently, $50 \%$ of the CAs have an unexplained etiology; however, some etiological factors have been reported, including genetic, nutritional, infectious, and/or environmental factors, among which maternal nutritional status is highlighted. ${ }^{10,11}$ Despite this, there are few epidemiological studies on the prevalence of CAs and the association between the body mass index (BMI) of pregnant women and the development of CAs. ${ }^{12-14}$ These studies include only overweight and obese pregnant women.
Thus, the prevalence of CAs in pregnant women with different nutritional profiles remains unknown. ${ }^{15-17}$

Research on maternal BMI and the risk of CAs is important to render assistance to healthcare systems in developing strategies for the prevention of CAs. ${ }^{4,18}$ In this context, the objective of the present study was to evaluate and compare the prevalence and association of structural CAs according to the maternal BMI.

\section{Methods}

\section{Type of Study}

The present observational cross-sectional study included pregnant women carrying fetuses with structural CAs at the Hospital das Clínicas of the Faculdade de Medicina of the Universidade Federal de Goiás Goiânia, state of Goiás, Brazil. Data were collected between November 2014 and January 2016 . The present study was approved by the research ethics committee of the Hospital das Clínicas.

\section{Study Population}

We have included pregnant women at a high-risk prenatal outpatient clinic with fetuses diagnosed with structural CAs through morphological ultrasonography. Pregnant women whose fetuses were not diagnosed with structural CAs, pregnant women who had ultrasound at or after 14 weeks of gestation or who did not remember their pregestational weight were excluded. The selected patients were submitted 
to a private interview in which they were informed about the stages of the study and signed the informed consent form.

\section{Data Collection}

The sociodemographic data of the pregnant women were collected during the interview through questionnaires. In addition, anthropometric (weight, height) and obstetric (gestational age; fetal gender; previous pregnancies; previous abortion history; children with previous CAs; family history of CAs; maternal DM; alcohol, illicit drug, and tobacco consumption, and teratogenic medication use during pregnancy) data were collected. Gestational age was based on the first obstetrical ultrasonography performed to confirm the gestation, performed up to the 14th week of gestation. The pregestational weight was self-reported by the pregnant women. The height of the pregnant women was measured using a mechanical beam medical scale (Medical Antropometrístico Mechanical Scale Welmy, São Paulo, Brazil) in meters. The BMI was calculated by dividing the weight of the pregnant women $(\mathrm{kg})$ by the square of their height $\left(\mathrm{m}^{2}\right)$. The method of Atalah et al ${ }^{19}$ was employed to classify the nutritional status of the pregnant women according to the gross BMI value for the gestational age for the first trimester, which is recommended by the Brazilian Ministry of Health. ${ }^{19,20}$ The pregnant women were categorized into four groups: low weight, adequate weight, overweight, and obesity. After the expected date of delivery, data on the evolution of the gestation and on the confirmation of fetal gender were obtained through telephone interviews with the pregnant women.

\section{Statistical Analysis}

The sample size was of 153 women, calculated based on a $5 \%$ of error and on a 95\% confidence level. Statistical analyses were performed using the software Stata/SE version 12.0 (Stata Corporation, College Station, TX). Differences between distributions in particular groups were also evaluated by the MannWhitney test and by the Fisher exact test. In prevalence ratio (PR) calculations, the reference category was the group with the lowest prevalence, considering a 95\% confidence interval (CI). Values of $p \leq 0.05$ were considered statistically significant.

\section{Results}

\section{Characteristics of the Study Sample}

A total of 223 pregnant women with fetuses diagnosed with CAs through ultrasonography participated in the present study. The mean gestational age at the time of diagnosis was 12.83 weeks (standard deviation $[S D]=1.16$ ). After the calculation of the BMI, the pregnant women were categorized into groups: low weight $(20.18 \% ; n=45)$, adequate weight (43.50\%; $n=97)$, overweight $(22.87 \% ; n=51)$, and obese (13.45\%; $n=30$ ) (-Table 1).

Most of the pregnant women were aged between 19 and 29 years old (58.74\%; 121/223) and were of white, brown, or indigenous ethnicity (79.82\%; 178/223). A significant difference in age was observed in pregnant women with low weight; a greater proportion of women were aged between 19 and 29 years old (25.19\%; 33/45; $p=0.021 ; \mathrm{PR}=3.02$;
95\% CI:1.24-7.37), and had a family history of CAs (30.00\%; $15 / 45 ; p=0.049 ; \mathrm{PR}=1.73 ; 95 \% \mathrm{CI}=1.01-2.95)$. Normal weight was not associated with $\mathrm{DM}(\mathrm{PR}=3.09$; $95 \% \mathrm{CI}$ $=1.07-8.88 ; p=0.008)$; however, obesity was positively associated with $\mathrm{DM} \quad(\mathrm{PR}=3.09 ; 95 \% \mathrm{CI}=1.33-7.20$; $p=0.003$ ) (-Table 1). No significant differences in previous abortion history, children with previous CAs, previous pregnancies, alcohol, illicit drug, or tobacco consumption, teratogenic medication use during pregnancy, and evolution of gestation were found among groups.

\section{Prevalence of Structural Congenital Anomalies}

Of the analyzed fetuses with CAs, 51.12\% (114/223) were males, and 48.88\% were females (109/223). - Table 2 presents the prevalence of fetal structural CAs in pregnant women with low and normal weight and in those who were overweight and obese. Ten types of structural CAs were detected, with central nervous system (CNS) (30.94\%; 69/ $223)$ and genitourinary system (23.77\%; 53/223) anomalies being the most prevalent.

Multiple CAs accounted for $17.49 \%$ (39/223) of the anomalies, with a statistical difference among the four groups analyzed. The bivariate analysis showed an association between low weight for the gestational age and absence of multiple anomalies in pregnant women. Normal weight was associated with the presence of multiple anomalies and absence of lymphatic system anomalies. Obese pregnant women had the highest prevalence of lymphatic system anomalies (46.15\%), and obesity was determined to be associated with lymphatic system anomalies.

\section{Description of Subtypes of Congenital Defects}

- Table 3 presents the prevalence of subtypes of congenital defects in pregnant women with low and adequate weight and in those who were overweight and obese. Hydronephrosis/ pyelectasis was the most prevalent anomaly in the study sample (11.66\%; 26/223). Renal dysplasia was the most prevalent defect in pregnant women with low gestational weight (20\%; 9/45), followed by acrania/anencephaly (11.11\%; 5/45), hydrocephalus (11.11\%; 5/45), hydronephrosis/pyelectasis (11.11\%; 5/45), and gastroschisis (11.11\%; 5/45). In pregnant women with adequate weight, hydronephrosis/pyelectasis had the highest prevalence rate (13.40\%; 13/97). In overweight pregnant women, CNS anomalies were prevalent; however, hydronephrosis/pyelectasis had the highest prevalence rate (11.76\%; 6/51). In obese pregnant women, cystic hygroma had the highest prevalence rate $(20 \% ; 6 / 30)$.

\section{Discussion}

Congenital anomalies represent epidemiological relevance because they result in mortality in $\sim 276,000$ newborns per year worldwide. ${ }^{10,11}$ Despite this, there are few studies that report the reality of the center-west region of Brazil.

In the present study, we have analyzed patients from a tertiary referral public hospital in the care of high-risk pregnant women in the center-west region of Brazil, and report a higher frequency of CNS anomalies (30.94\%; 69/ 


\begin{tabular}{|c|c|c|c|c|c|c|c|c|c|c|c|c|c|c|c|c|c|c|c|c|c|c|c|c|}
\hline & 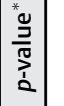 & & $\begin{array}{l}\text { to } \\
0 \\
0 \\
0 \\
0\end{array}$ & & & & $\stackrel{\substack{n \\
0}}{\circ}$ & & & 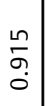 & & & $\begin{array}{l}\text { F. } \\
\stackrel{2}{2} \\
\stackrel{2}{0}\end{array}$ & & & $\begin{array}{l}\tilde{N} \\
\hat{\sigma} \\
0\end{array}$ & & & $\begin{array}{l}\infty \\
\stackrel{\infty}{0} \\
0 \\
0\end{array}$ & & & $\left|\begin{array}{l}0 \\
0 \\
0 \\
0\end{array}\right|$ & & \\
\hline & $\begin{array}{l}\overline{\bar{\sigma}} \\
\stackrel{\circ}{ } \\
\stackrel{\alpha}{\sigma} \\
\stackrel{\alpha}{\alpha}\end{array}$ & 1 & & 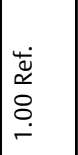 & 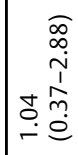 & 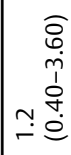 & & 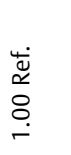 & 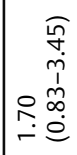 & & 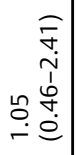 & 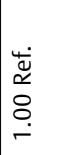 & & 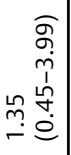 & 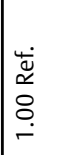 & & 这 & 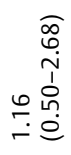 & & 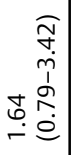 & 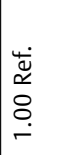 & & 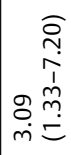 & 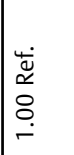 \\
\hline $\begin{array}{l}\vec{z} \\
\bar{y} \\
0 \\
0\end{array}$ & $\stackrel{\widehat{巳}}{=}$ & 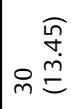 & & 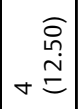 & 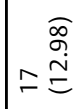 & o & & $\overline{\bar{N}} \stackrel{\widehat{D}}{\bar{\Xi}}$ & 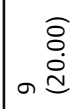 & & 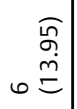 & 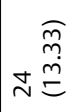 & & $\begin{aligned} & \widehat{\Omega} \\
0 & \stackrel{0}{E}\end{aligned}$ & 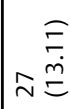 & & 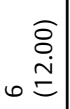 & $\underset{\substack{\infty \\
\infty}}{\stackrel{\widehat{N}}{\mathscr{n}}}$ & & $\begin{aligned} & \widehat{\tilde{m}} \\
\overline{\dot{b}} & \bar{v}\end{aligned}$ & $\begin{array}{r}\frac{D}{\sigma} \\
\sigma \stackrel{\sigma}{\sigma}\end{array}$ & & 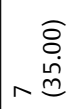 & 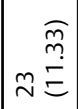 \\
\hline & 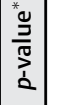 & & $\begin{array}{l}\stackrel{*}{*} \\
\stackrel{1}{0} \\
0 \\
0\end{array}$ & & & & 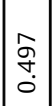 & & & 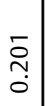 & & & 列 & & & $\begin{array}{l}0 \\
: \\
0 \\
0\end{array}$ & & & $\frac{\bar{\sigma}}{\sigma}$ & & & $\left|\begin{array}{l}0 \\
\stackrel{2}{2} \\
0 \\
0\end{array}\right|$ & & \\
\hline$\neq$ & 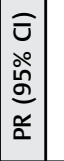 & 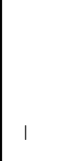 & & 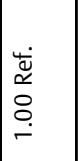 & 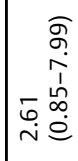 & 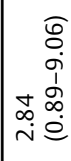 & & 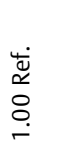 & 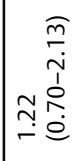 & & 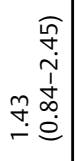 & 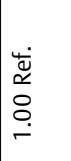 & & 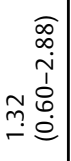 & 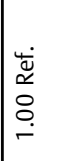 & & & 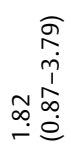 & & 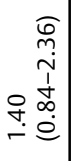 & 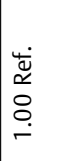 & & 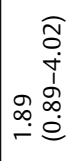 & 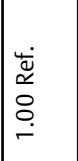 \\
\hline 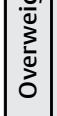 & 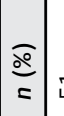 & 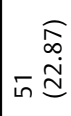 & & 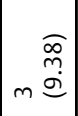 & 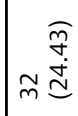 & 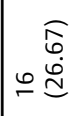 & & m) & \begin{aligned} & \multirow{6}{0}{} \\
$\simeq & \stackrel{d}{d}\end{aligned}$ & & 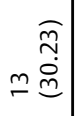 & $\underset{m}{\stackrel{\Xi}{\check{I}}}$ & & n $\begin{array}{c}\overline{\bar{d}} \\
\stackrel{\bar{d}}{ \pm}\end{array}$ & 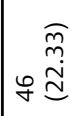 & & $\begin{array}{r}\widehat{\Xi} \\
\wedge \\
\stackrel{\dot{\Xi}}{\Xi}\end{array}$ & 将 & & 鿖 & $\therefore \stackrel{\substack{+\infty \\
=}}{\stackrel{\infty}{\infty}}$ & & 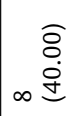 & भ \\
\hline & $\mid$ & & $\mid \begin{array}{l}\hat{a} \\
\hat{0} \\
0\end{array}$ & & & & $\begin{array}{c}0 \\
\stackrel{\infty}{0} \\
\stackrel{0}{0}\end{array}$ & & & 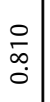 & & & $\begin{array}{l}\infty \\
\stackrel{\infty}{h} \\
0 \\
0\end{array}$ & & & $\begin{array}{l}\text { ñ } \\
\hat{o} \\
0\end{array}$ & & & $\begin{array}{l}\hat{\infty} \\
\stackrel{\infty}{0} \\
0\end{array}$ & & & $\mid \begin{array}{l}* \\
0 \\
0 \\
0 \\
0\end{array}$ & & \\
\hline $\begin{array}{l}\text { 喜 } \\
\frac{9}{0} \\
\frac{0}{3}\end{array}$ & 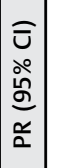 & 1 & & 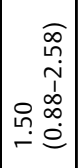 & 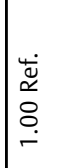 & 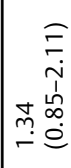 & & 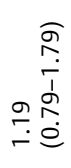 & 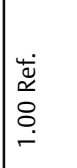 & & 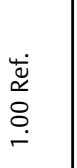 & 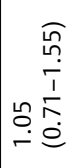 & & 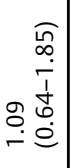 & 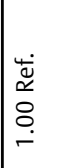 & & 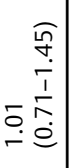 & 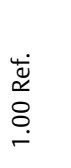 & & 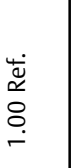 & 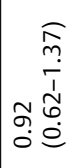 & & 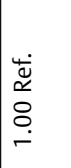 & 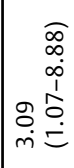 \\
\hline 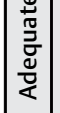 & $\stackrel{\frac{\$}{\circ}}{=}$ & 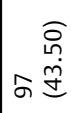 & & 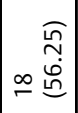 & 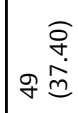 & 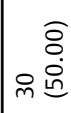 & & 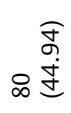 & 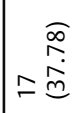 & & 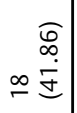 & 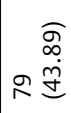 & & $\begin{array}{r}\mathscr{Q} \\
\stackrel{\dot{\dot{\Xi}}}{\infty}\end{array}$ & 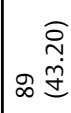 & & 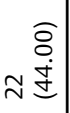 & 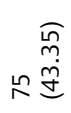 & & 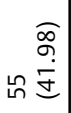 & 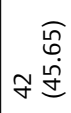 & & m & \\
\hline & 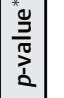 & & 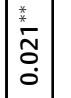 & & & & $\begin{array}{c}\hat{\infty} \\
\tilde{m} \\
0\end{array}$ & & & $\stackrel{\stackrel{\infty}{\leftrightarrow}}{\stackrel{0}{0}}$ & & & 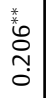 & & & $\begin{array}{l}\text { gे } \\
\text { d. }\end{array}$ & & & $\begin{array}{l}u \\
\stackrel{0}{0} \\
0\end{array}$ & & & $\mid \begin{array}{l}7 \\
0 \\
m \\
m \\
0\end{array}$ & & \\
\hline \pm & 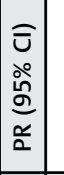 & | & & 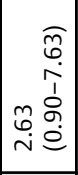 & 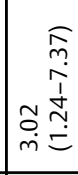 & & & 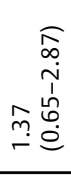 & 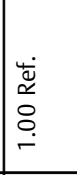 & & 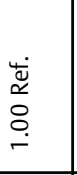 & 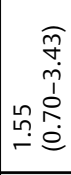 & & 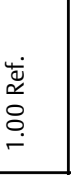 & 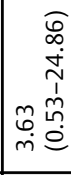 & & 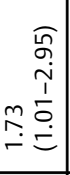 & 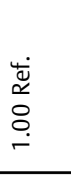 & & & 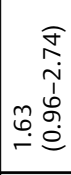 & & 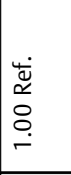 & 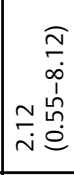 \\
\hline $\mid \begin{array}{l}\cdot \overline{\bar{\sigma}} \\
3 \\
3 \\
0 \\
-1\end{array}$ & $\stackrel{\overparen{9}}{=}$ & 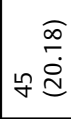 & & 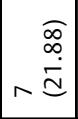 & m $\underset{m}{\stackrel{\sigma}{\stackrel{a}{g}}}$ & $\underset{\substack{\widehat{m} \\
\text { n }}}{\infty}$ & & 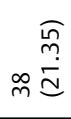 & r & & $\begin{array}{r}\widehat{\Omega} \\
\stackrel{\Omega}{m} \\
0\end{array}$ & $\begin{aligned} & \underset{6}{0} \\
m & \stackrel{\dot{d}}{d}\end{aligned}$ & & $\begin{array}{r}\widehat{\infty} \\
\infty \\
-\stackrel{\infty}{\mathscr{\omega}} \\
\end{array}$ & J & & 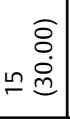 & $\stackrel{\stackrel{f}{m}}{\stackrel{\text { f }}{=}}$ & & $\begin{aligned} & \widehat{\tilde{m}} \\
\overline{0} & \stackrel{\dot{b}}{=}\end{aligned}$ & 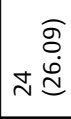 & & v & $\underset{\gamma}{\stackrel{\infty}{\stackrel{\infty}{\Sigma}}}$ \\
\hline $\begin{array}{l}\overline{\overrightarrow{0}} \\
\stackrel{0}{\circ}\end{array}$ & 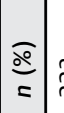 & 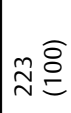 & & 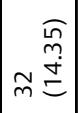 & 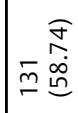 & $\begin{array}{r}\widehat{\bar{g}} \\
\stackrel{\stackrel{d}{d}}{\circ}\end{array}$ & & 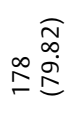 & 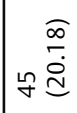 & & 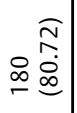 & 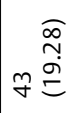 & & $=\underset{\widehat{S}}{\stackrel{\Xi}{\leftrightarrows}}$ & 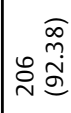 & & $\begin{array}{r}\widehat{\widetilde{J}} \\
\stackrel{\mathbb{Z}}{\mathbb{Z}}\end{array}$ & 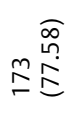 & & 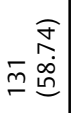 & 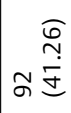 & & 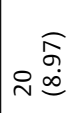 & $\begin{array}{l}\widehat{\hat{m}} \\
\stackrel{0}{a} \\
\stackrel{\alpha}{\sigma}\end{array}$ \\
\hline 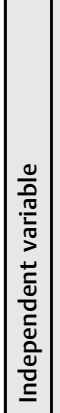 & & 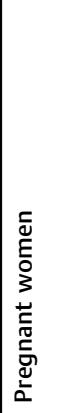 & 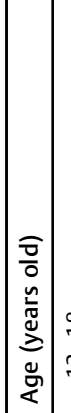 & 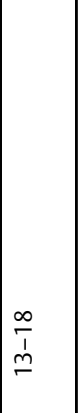 & $\begin{array}{l}\stackrel{2}{\sim} \\
\stackrel{\sigma}{\sigma}\end{array}$ & 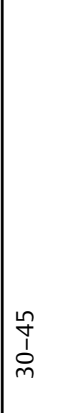 & 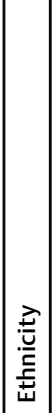 & 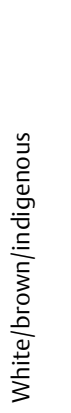 & 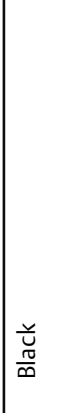 & 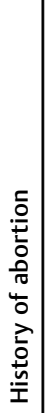 & $\stackrel{\check{\nu}}{\nu}$ & $\frac{0}{2}$ & 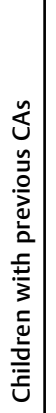 & $\stackrel{\check{\nu}}{\check{\nu}}$ & $\frac{0}{2}$ & 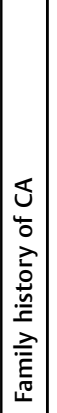 & $\check{\check{y}}$ & $\frac{0}{2}$ & 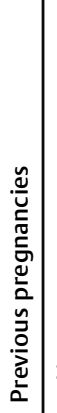 & 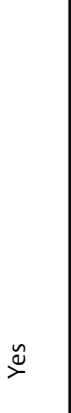 & zo & 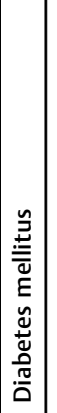 & $\stackrel{\Xi}{\nu}$ & i \\
\hline
\end{tabular}




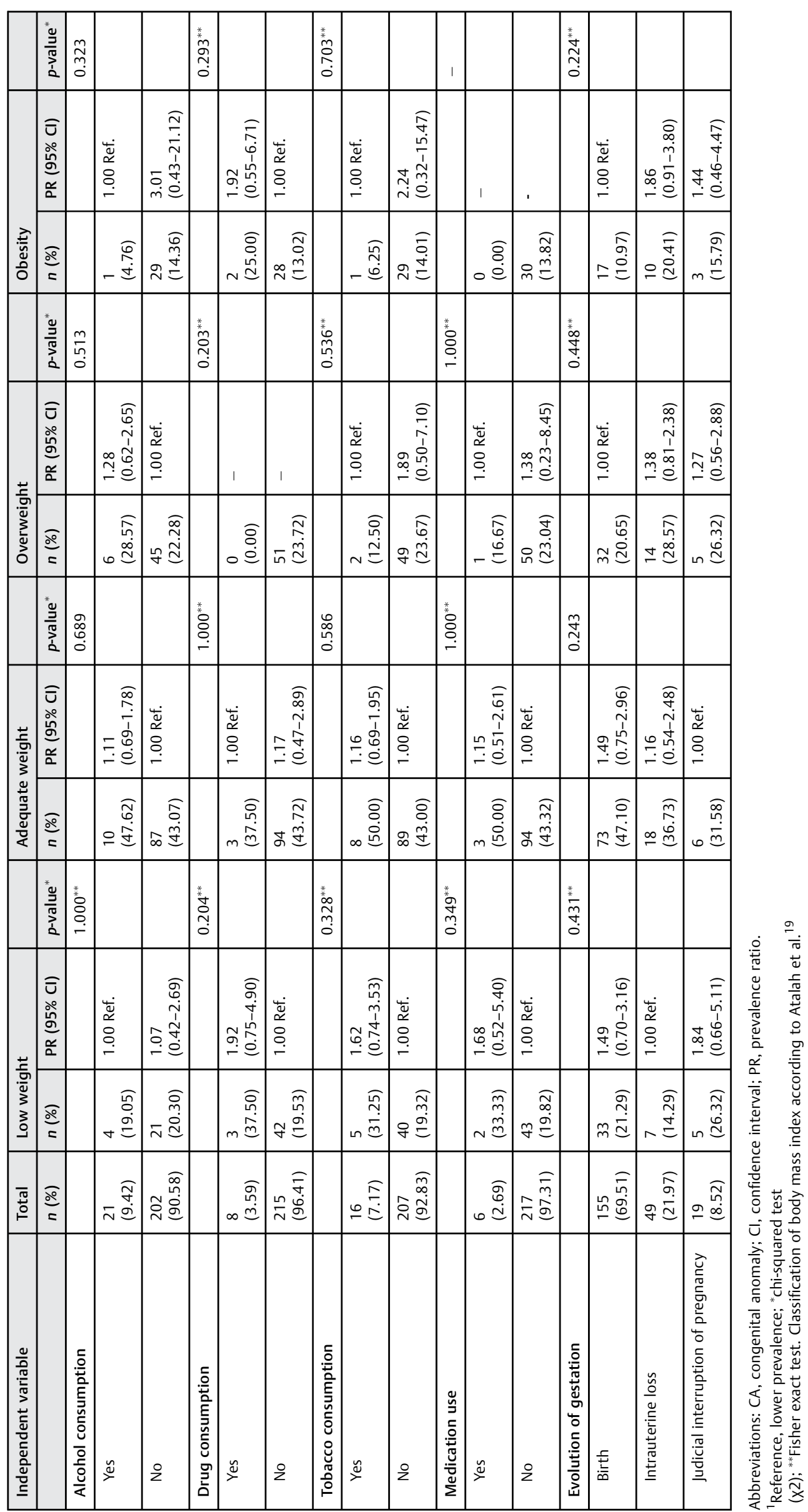




\begin{tabular}{|c|c|c|c|c|c|c|c|c|c|c|c|c|c|c|c|c|c|c|c|c|c|c|c|c|c|}
\hline & 遂 & $\begin{array}{c}0 \\
0 \\
\vdots \\
0\end{array}$ & & & $\begin{array}{l}\tilde{1} \\
0 \\
0 \\
0\end{array}$ & & & $\mid \begin{array}{l}0 \\
0 \\
0 \\
0 \\
0\end{array}$ & & & | & & & 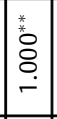 & & & $\begin{array}{l}8 \\
0 \\
0 \\
0\end{array}$ & & & 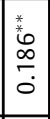 & & & 善 & & \\
\hline & 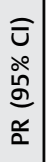 & & 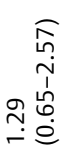 & 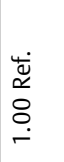 & & 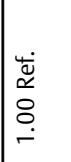 & 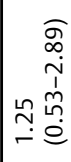 & & 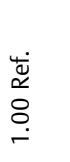 & 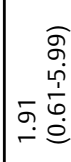 & & 1 & 1 & & & 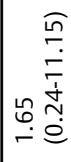 & & 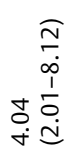 & 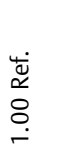 & & 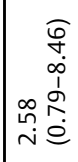 & 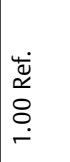 & & 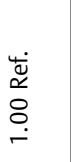 & 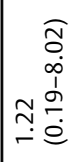 \\
\hline 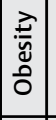 & 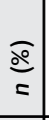 & & 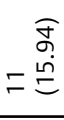 & 욜 & & 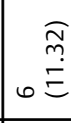 & 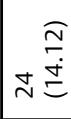 & & $m \stackrel{\widehat{Q}}{\stackrel{\leftrightarrow}{\Xi}}$ & 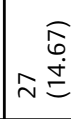 & & $\begin{array}{l}\overline{8} \\
0 \\
0\end{array}$ & 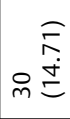 & & $\begin{array}{r}\widehat{m} \\
\stackrel{m}{0} \\
-\infty\end{array}$ & 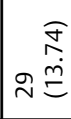 & & 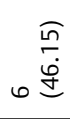 & $\stackrel{\widehat{\widetilde{f}}}{\stackrel{\Im}{\Xi}}$ & & $\underset{\substack{m \\
m \\
\sim}}{\stackrel{m}{m}}$ & 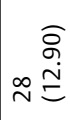 & & $\begin{array}{r}E \\
-E \\
\end{array}$ & શ \\
\hline & \pm & 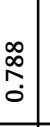 & & & 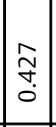 & & & $\mid \begin{array}{l}n \\
\hat{\sigma} \\
o \\
0\end{array}$ & & & 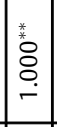 & & & 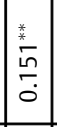 & & & 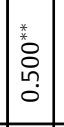 & & & 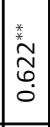 & & & $\begin{array}{l}0 \\
0 \\
\infty \\
0 \\
0 \\
0 \\
0\end{array}$ & & \\
\hline & 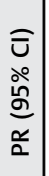 & & & 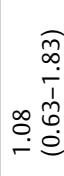 & & 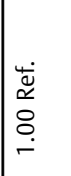 & 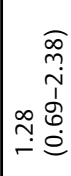 & & 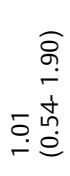 & 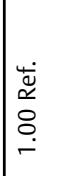 & & 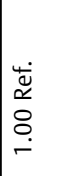 & 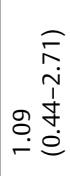 & & 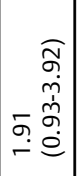 & 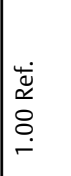 & & 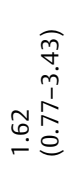 & 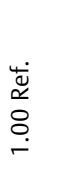 & & 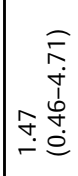 & 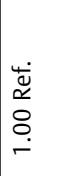 & & 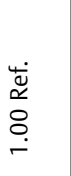 & 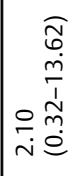 \\
\hline $\begin{array}{l}\bar{\sigma} \\
\overline{\bar{v}} \\
\overline{0} \\
0 \\
0\end{array}$ & 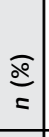 & & 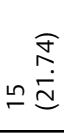 & 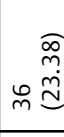 & & 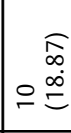 & 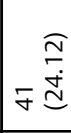 & & 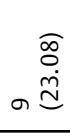 & 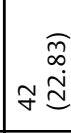 & & 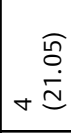 & 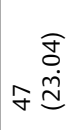 & & $\begin{array}{r}\widehat{E} \\
\text { 的立 }\end{array}$ & 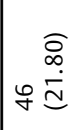 & & $\begin{array}{r}\widehat{R} \\
\stackrel{i}{0} \\
+\stackrel{0}{0}\end{array}$ & 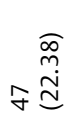 & & 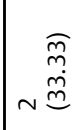 & 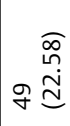 & & 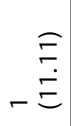 & in \\
\hline & 足 & 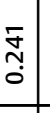 & & & $\begin{array}{c}\infty \\
\stackrel{\infty}{\Upsilon} \\
0\end{array}$ & & & $\begin{array}{l}0 \\
\vdots \\
0 \\
\end{array}$ & & & $\begin{array}{l}\tilde{N} \\
\hat{0} \\
0 \\
\end{array}$ & & & 䓵 & & & $\mid \begin{array}{l}0 \\
\tilde{z} \\
\tilde{y} \\
0 \\
0\end{array}$ & & & $\begin{array}{l}0 \\
0 \\
0 \\
0 \\
0 \\
0\end{array}$ & & & $\begin{array}{l}n \\
\\
0 \\
0\end{array}$ & & \\
\hline $\begin{array}{c} \\
+\frac{5}{5} \\
\frac{0}{0} \\
\frac{3}{3}\end{array}$ & $\begin{array}{l}\overline{\bar{c}} \\
\stackrel{\circ}{\circ} \\
\stackrel{\alpha}{\alpha} \\
\frac{\alpha}{\alpha}\end{array}$ & & 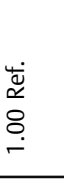 & 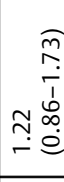 & & 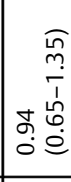 & 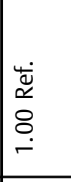 & & 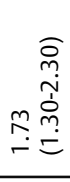 & 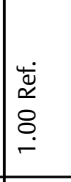 & & 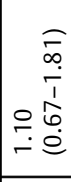 & 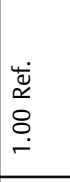 & & 芯 & 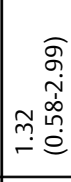 & & 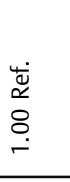 & 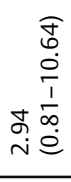 & & 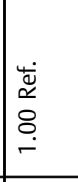 & 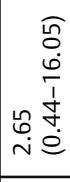 & & 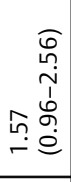 & 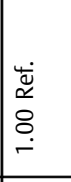 \\
\hline $\begin{array}{l}\frac{\tilde{y}}{0} \\
\frac{0}{0} \\
\frac{\vec{v}}{<}\end{array}$ & $\stackrel{\frac{\Phi}{\Phi}}{=}$ & & 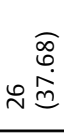 & $\therefore$ 일 & & 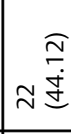 & 贶 & & 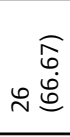 & 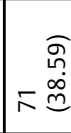 & & 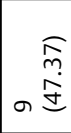 & 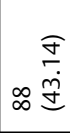 & & $+\underset{\substack{m \\
\stackrel{m}{m}}}{+\stackrel{c}{m}}$ & 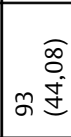 & & $\sim \stackrel{\substack{\infty \\
\stackrel{n}{n}}}{=}$ & 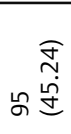 & & 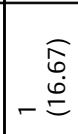 & প. & & 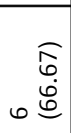 & б) \\
\hline & 要 & $\begin{array}{c}0 \\
\stackrel{0}{0} \\
0\end{array}$ & & & 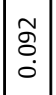 & & & $\mid \begin{array}{l}* \\
\tilde{z} \\
0 \\
0\end{array}$ & & & $\begin{array}{l}\frac{0}{9} \\
\stackrel{0}{\circ}\end{array}$ & & & 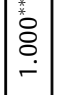 & & & 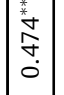 & & & 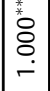 & & & $\begin{array}{l}- \\
\vdots \\
0 \\
0 \\
0\end{array}$ & & \\
\hline & 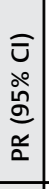 & & 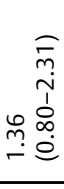 & 文 & & 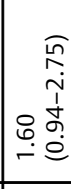 & 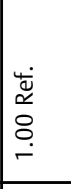 & & 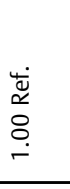 & 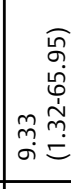 & & 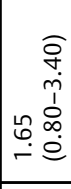 & 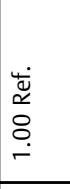 & & & 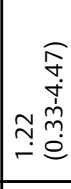 & & 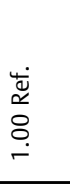 & 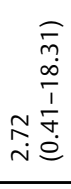 & & 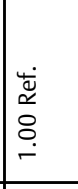 & 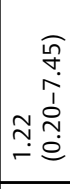 & & 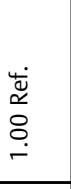 & 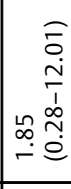 \\
\hline 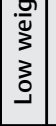 & 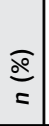 & & 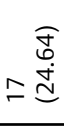 & $\stackrel{\infty}{\stackrel{\infty}{\infty}} \stackrel{\infty}{=}$ & & 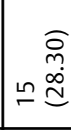 & 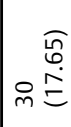 & & 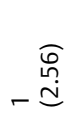 & 家 & & o & 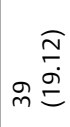 & & 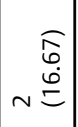 & 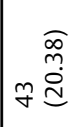 & & $\begin{array}{r}\hat{\sigma} \\
-\stackrel{5}{=}\end{array}$ & 芯 & & $\begin{array}{r}\widehat{E} \\
-\stackrel{6}{0} \\
-\end{array}$ & 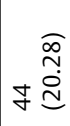 & & 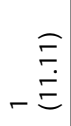 & 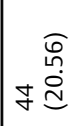 \\
\hline & ๑े & & $\begin{array}{l}\text { oे } \\
\text { के }\end{array}$ & $\begin{array}{l}\stackrel{8}{0} \\
\dot{0}\end{array}$ & & 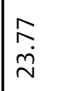 & 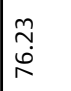 & & 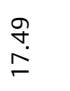 & 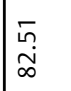 & & $\begin{array}{l}\tilde{n} \\
\infty \\
\infty\end{array}$ & 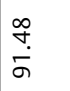 & & 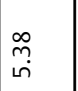 & \begin{tabular}{l}
$\tilde{\sigma}$ \\
\multirow{\sigma}{*}{}
\end{tabular} & & $\begin{array}{l}\stackrel{m}{\infty} \\
\stackrel{\leftrightarrow}{i}\end{array}$ & $\begin{array}{l}\text { Fे } \\
\dot{\sigma}\end{array}$ & & 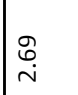 & $\begin{array}{l}\bar{m} \\
\stackrel{n}{a}\end{array}$ & & $\begin{array}{l}\text { ¿े } \\
\dot{+}\end{array}$ & 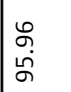 \\
\hline $\begin{array}{l}\text { 苛 } \\
\stackrel{0}{\circ}\end{array}$ & $=$ & & 8 & 望 & & nิ & $\stackrel{?}{\stackrel{2}{2}}$ & & g & 㫣 & & 9 & ণ্ণ & & $\simeq$ & $\overline{\bar{N}}$ & & $\stackrel{m}{m}$ & $\stackrel{\circ}{N}$ & & 0 & $\stackrel{N}{N}$ & & $\sigma$ & $\stackrel{\vec{N}}{\sim}$ \\
\hline 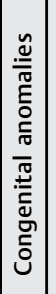 & & 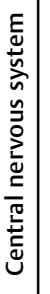 & $\stackrel{\check{\nu}}{\stackrel{\Perp}{\nu}}$ & 2 & 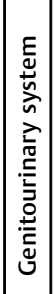 & $\stackrel{y}{\check{\nu}}$ & $\stackrel{0}{2}$ & 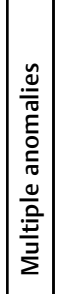 & $\stackrel{\check{\nu}}{\nu}$ & 2 & 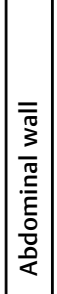 & $\stackrel{\check{\nu}}{\rightleftharpoons}$ & 2 & 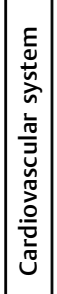 & $\stackrel{\breve{~}}{\rightleftharpoons}$ & $\stackrel{0}{2}$ & 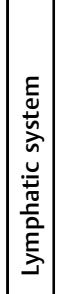 & $\stackrel{\check{\nu}}{\check{\nu}}$ & z & 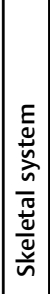 & $\stackrel{\tilde{y}}{\nu}$ & 2 & $\begin{array}{l}\mathbb{\Xi} \\
\tilde{\Phi}\end{array}$ & 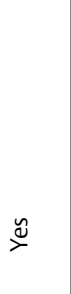 & z \\
\hline
\end{tabular}




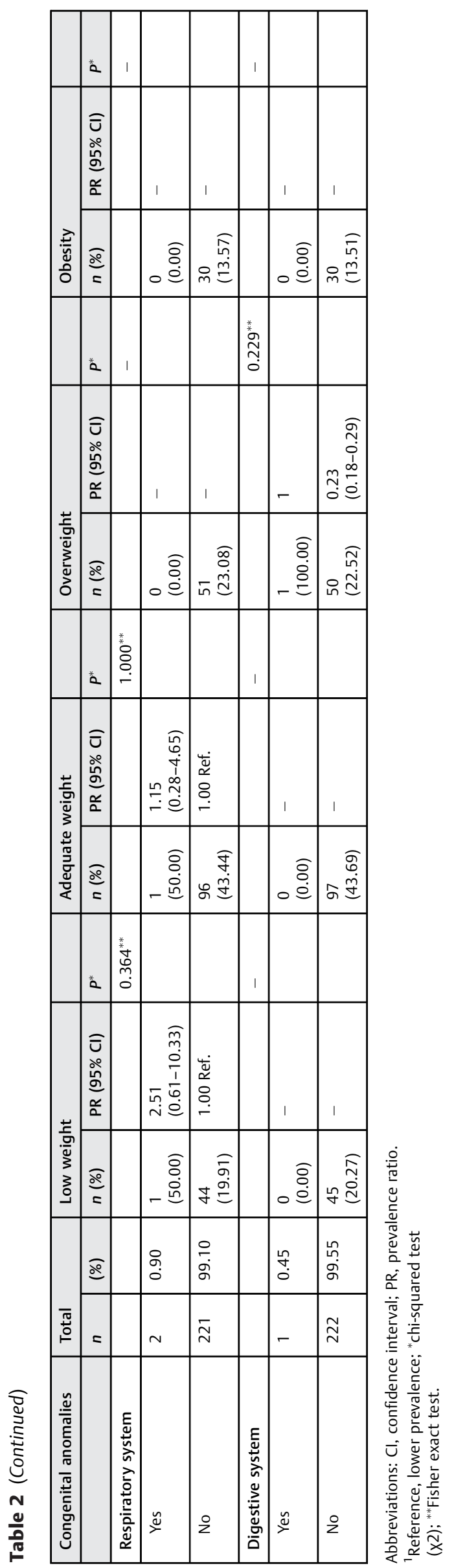

223), followed by genitourinary system (23.77\%; 53/223), and multiple CAs (17.49\%; 39/223). Indian studies showed similar results. ${ }^{21-23}$

The etiology of CNS malformations is multifactorial, involving complex interactions between genetic and environmental factors, constituting one of the most common congenital defects..$^{22,24,25}$ Sunitha et al ${ }^{22}$ analyzed 360 pregnant women with fetuses presenting structural abnormalities, and also observed a higher frequency of CNS anomalies (37\%), followed by genitourinary system abnormalities $(20 \%)$ and multiple CAs (11\%). In addition, other studies have shown the higher prevalence of genitourinary system malformations and of genitourinary system malformations..$^{21,23}$

It is known that $50 \%$ of the CAs may have an unknown etiology that can be attributed to genetic and environmental factors, including maternal nutritional aspects. ${ }^{10,11}$ This etiological factor has become relevant due the drastic change in the demographics of pregnant women in the last decade, with a higher number of overweight or obese women at conception being observed. ${ }^{26}$ In the present study, this phenomenon still cannot be observed, since the frequency of obese pregnant women was lower than the frequency of pregnant women of adequate weight. The study population of the present study comes from a tertiary service; therefore, it is likely that, in the analysis of the general population of pregnant women, the frequency of pregnant women who are overweight and obese is higher than the one found here. However, there is a tendency of these data to be altered in future studies. The results of the present study indicated an association between pregnant women with adequate weight and the presence of multiple CAs, and among obese pregnant women with the presence of anomalies of the lymphatic system.

The presence of multiple CAs, in any category of BMI, may be explained by the higher consumption of alcohol, tobacco, and of teratogenic medications during pregnancy in the present study. Although no statistical difference was observed between the groups and the consumption of teratogenic substances, we have noticed that pregnant women with adequate weight consumed more teratogenics than the other groups. The higher consumption of teratogenic substances by this group may have contributed to a higher prevalence of anomalies. However, the comparability of these results with those of national and international studies is limited, mainly because most studies dealing with CAs and gestational BMI have focused more on obese pregnant women. ${ }^{17,27-29}$

Lifestyle recommendations for couples planning to have children and guidelines for the cessation of smoking, of alcohol consumption, and of the use of illicit drugs, which are teratogenic substances, currently exist. ${ }^{18,30}$ However, it is necessary to reinforce the awareness of the population regarding the harm of teratogenics during pregnancy. Teratogenic substances can cause clinical manifestations such as abortion, CAs, intrauterine growth retardation, and mental deficiency. ${ }^{30-32}$

Multiple CAs represent a serious category of structural defects and are associated with high rates of stillbirth, preterm birth, and low birthweight. ${ }^{33}$ They are usually related to genetic syndromes and are a part of a complex group of anomalies with lethal cumulative effects in the 
Prevalence and Association of Congenital Anomalies Moraes et al. 287

Table 3 Prevalence of subtypes of congenital anomalies in pregnant women attending a high-risk prenatal outpatient clinic from 2014 to 2016 according to body mass index

\begin{tabular}{|c|c|c|c|c|c|}
\hline Congenital anomalies & Low weight & Adequate weight & Overweight & Obesity & Total \\
\hline & $n(\%)$ & $n(\%)$ & $n(\%)$ & $n(\%)$ & $n(\%)$ \\
\hline \multicolumn{6}{|l|}{ Central nervous system } \\
\hline Acrania/Anencephaly & $5(11.11)$ & $6(6.19)$ & $5(9.80)$ & $3(10.00)$ & $19(8.52)$ \\
\hline Spina bifida/meningocele & $3(6.67)$ & $5(5.15)$ & $2(3.92)$ & $0(0.0)$ & $10(4.48)$ \\
\hline Hydranencephaly & $0(0.0)$ & $0(0.0)$ & $3(5.88)$ & $0(0.0)$ & $3(1.35)$ \\
\hline Hydrocephalus & $5(11.11)$ & $9(9.28)$ & $4(7.84)$ & $5(16.67)$ & $23(10.31)$ \\
\hline Holoprosencephaly & $2(4.44)$ & $3(3.09)$ & $0(0.0)$ & $1(3.33)$ & $6(2.69)$ \\
\hline Others & $2(4.44)$ & $3(3.09)$ & $1(1.96)$ & $2(6.67)$ & $8(3.59)$ \\
\hline \multicolumn{6}{|l|}{ Genitourinary system } \\
\hline Renal dysplasia & $9(20.00)$ & $5(5.15)$ & $3(5.88)$ & $3(10.00)$ & $20(8.97)$ \\
\hline Hydronephrosis/pyelectasis & $5(11.11)$ & $13(13.40)$ & $6(11.76)$ & $2(6.67)$ & $26(11.66)$ \\
\hline Megacystis & $1(2.22)$ & $3(3.09)$ & $1(1.96)$ & $0(0.0)$ & $5(2.24)$ \\
\hline Obstructive uropathy & $0(0.0)$ & $1(1.03)$ & $0(0.0)$ & $1(3.33)$ & $2(0.90)$ \\
\hline \multicolumn{6}{|l|}{ Multiple congenital anomalies } \\
\hline Craniofacial + cardiac & $1(2.22)$ & $7(7.22)$ & $3(5.88)$ & $1(3.33)$ & $12(5.38)$ \\
\hline Craniofacial + digestive & $0(0.0)$ & $2(2.06)$ & $2(3.92)$ & $1(3.33)$ & $5(2.24)$ \\
\hline Craniofacial + renal & $0(0.0)$ & $3(3.09)$ & $2(3.92)$ & $0(0.0)$ & $5(2.24)$ \\
\hline Digestive + renal & $0(0.0)$ & $3(3.09)$ & $0(0.0)$ & $1(3.33)$ & $4(1.79)$ \\
\hline Craniofacial + members & $0(0.0)$ & $11(11.34)$ & $2(15.4)$ & $0(0.0)$ & $13(5.83)$ \\
\hline \multicolumn{6}{|l|}{ Abdominal wall } \\
\hline Gastroschisis & $5(11.11)$ & $6(6.19)$ & $4(7.84)$ & $0(0.0)$ & $15(6.73)$ \\
\hline Diaphragmatic hernia & $0(0.0)$ & $2(2.06)$ & $0(0.0)$ & $0(0.0)$ & $2(0.90)$ \\
\hline Omphalocele & $1(2.22)$ & $1(1.03)$ & $0(0.0)$ & $0(0.0)$ & $2(0.90)$ \\
\hline \multicolumn{6}{|l|}{ Cardiovascular system } \\
\hline Bradyarrhythmia & $0(0.0)$ & $2(2.06)$ & $2(3.92)$ & $0(0.0)$ & $4(1.79)$ \\
\hline Cardiomegaly & $1(2.22)$ & $2(2.06)$ & $2(3.92)$ & $0(0.0)$ & $5(2.24)$ \\
\hline Others & $1(2.22)$ & $0(0.0)$ & $1(1.96)$ & $1(3.33)$ & $3(1.35)$ \\
\hline \multicolumn{6}{|l|}{ Lymphatic system } \\
\hline Cystic hygroma & $1(7.7)$ & $2(2.06)$ & $4(7.84)$ & $6(20.00)$ & $13(5.83)$ \\
\hline \multicolumn{6}{|l|}{ Skeletal system } \\
\hline Thanatophoric dwarfism & $0(0.0)$ & $1(1.03)$ & $1(1.96)$ & $0(0.0)$ & $2(0.90)$ \\
\hline Osteogenesis imperfecta & $0(0.0)$ & $0(0.0)$ & $1(1.96)$ & $0(0.0)$ & $1(0.45)$ \\
\hline Achondroplasia & $1(2.22)$ & $0(0.0)$ & $0(0.0)$ & $2(6.67)$ & $1(0.45)$ \\
\hline \multicolumn{6}{|l|}{ Face } \\
\hline Cleft palate & $1(2.22)$ & $2(2.06)$ & $0(0.0)$ & $1(3.33)$ & $4(1.79)$ \\
\hline Cleft lip & $0(0.0)$ & $4(4.12)$ & $1(1.96)$ & $0(0.0)$ & $5(2.24)$ \\
\hline \multicolumn{6}{|l|}{ Respiratory system } \\
\hline $\begin{array}{l}\text { Pulmonary cystic adenomatoid } \\
\text { malformation }\end{array}$ & $1(2.22)$ & $1(1.03)$ & $0(0.0)$ & $0(0.0)$ & $2(0.90)$ \\
\hline \multicolumn{6}{|l|}{ Digestive system } \\
\hline $\begin{array}{l}\text { Atresia of the second portion } \\
\text { of the duodenum }\end{array}$ & $0(0.0)$ & $0(0.0)$ & $1(1.96)$ & $0(0.0)$ & $1(0.45)$ \\
\hline Total & $45(100.00)$ & $97(100.00)$ & $51(100.00)$ & $30(100.00)$ & $223(100.00)$ \\
\hline
\end{tabular}


intrauterine period. ${ }^{34}$ Moreover, pregnant women with normal weight had a higher prevalence of intrauterine loss and a of a family history of CAs, which may be related to a genetic predisposition. $^{35,36}$ Although no genetic study was performed, this factor may have contributed to a higher prevalence of multiple CAs and of intrauterine losses.

In the present study, the frequency of obese pregnant women was lower than the frequency of pregnant women of adequate weight. Similar studies indicated that the detection rate for anomalies was lower in obese pregnant women. ${ }^{13,37,38}$ Excessive abdominal adipose tissue is associated with significant limitations in the assessment of fetal anatomy using ultrasonography in the $1^{\text {st }}$ and $2^{\text {nd }}$ trimesters of pregnancy. $^{13,14,37,39}$ This, perhaps, is the factor that has contributed to a reduced sample of obese pregnant women in the present study. Therefore, obese pregnant women should be advised about the risks of inadequate visualization during fetal ultrasonography, requiring more follow-up during the pregnancy. ${ }^{39}$

The literature indicates that maternal obesity is associated with neural tube defects, including hydrocephalus, cardiac defects, renal cysts, orofacial clefts, anorectal atresia, limb reduction anomalies, omphalocele, and diaphragmatic hernia. ${ }^{16,17,28,40}$ However, obese pregnant women had a significantly high prevalence of lymphatic system anomalies, due to cystic hygroma, and it is important to note that no reports on this association exist in the literature. Lymphatic system anomalies may be associated with chromosomal disorders, and the morbidity of these lesions is dependent on their location. ${ }^{41}$ If cystic hygroma does not regress until the $18^{\text {th }}$ week of gestation, the fetus could possibly have chromosomal or nonchromosomal anomalies, with a probability of $>90 \% .{ }^{42}$

Studies have shown that folic acid deficiency can cause changes in DNA synthesis and chromosomal alterations, and that excessive maternal adipose tissue interferes with the folate metabolism. ${ }^{43,44}$ The risk of neural tube defects in the offspring has also been reported. ${ }^{43}$ However, it was not possible to infer that fetuses of obese pregnant women developed lymphatic system anomalies owing to the interference of adipose tissue with folic acid metabolism.

Overweight and obese pregnant women had a higher prevalence of DM, and an association between obesity and DM was observed. It is hypothesized that hyperglycemia impairs the development of the vitelline sac and of the placenta through increased production and release of oxygen free radicals and inositol and arachidonic acid deficiency, which induce a reduction in placental communication between the pregnant woman and her fetus. ${ }^{45,46}$ Uncontrolled hyperglycemia in the first weeks of gestation causes severe complications such as the risk of miscarriage and CAs, including atrial septal defect, anencephaly, sacral and adrenal agenesis. In the $2^{\text {nd }}$ trimester, maternal hyperglycemia causes exacerbated fetal growth and increased risk of fetal death during the last 4 to 6 weeks of gestation. ${ }^{47}$ In this case, inadequate metabolic monitoring during organogenesis is considered the main factor associated with the development of CAs. ${ }^{45}$ The literature and our findings reinforce the importance of clarifying the severity of DM and its role in the alteration of obstetric parameters and in the development of CAs in fetuses of overweight and of obese pregnant women during prenatal visits. $^{48,49}$

The present study has some limitations. First, performing fetal genetic tests was impossible, as they were not available for the patients in our study population who visited a public outpatient clinic. The genetic evaluation would have better clarified the anomalies detected. Second, the estimation of the sample size was not performed for abnormalities in the different systems, which may compromise the association of gestational BMI classifications, according to the system or subtypes of CAs. Third, the newborns in the present study were not evaluated for early or late neonatal mortality, and vitamin deficiencies were not evaluated in the pregnant women and in the newborns.

However, as a positive point, the present study presents the reality of a tertiary center in the center-west region of Brazil, the stratification of the prevalence of CAs in pregnant women with different BMIs considering the four groups, since some studies unify the groups of overweight and obese pregnant women, as well as the sample number, and the follow-up of the patients until the birth of the fetus.

\section{Conclusion}

In conclusion, the present study demonstrated that there are differences between the profiles of CAs in the groups of pregnant women according to the maternal BMI. Obese pregnant women had more abnormalities of the lymphatic system. In addition, it has also been verified that multiple CAs, generally caused by genetic defects, are independent of lower or higher maternal weight. In this case, it is observed that genetic counseling should be made available to all couples in order to prevent certain malformations. These findings reinforce the need for the identification and screening of risk factors for CAs, regardless of BMI, as well as the importance of discussing with pregnant women maternal nutrition and its effect on fetal development and on neonatal outcome. The implementation of public policies is needed to have more planned pregnancies, and genetic testing in the public health system can contribute to the optimization of the diagnosis of CAs and may elucidate the association between CAs and maternal BMI.

\section{Contributors}

All of the authors contributed with the project and data interpretation, the writing of the article, the critical review of the intellectual content, and with the final approval of the version to be published.

\section{Conflicts of Interest}

The authors have no conflicts of interest to declare.

\section{Acknowledgments}

We appreciate the financial support from Coordenação de Aperfeiçoamento de Pessoal de Nível Superior (CAPES) for providing a PhD scholarship for Melo N. C. and Moraes L. C. 


\section{References}

1 Dean SV, Lassi ZS, Imam AM, Bhutta ZA. Preconception care: nutritional risks and interventions. Reprod Health 2014;11 (Suppl 3):S3. Doi: 10.1186/1742-4755-11-S3-S3

2 Sato AP, Fujimori E. Nutritional status and weight gain in pregnant women. Rev Lat Am Enfermagem 2012;20(03):462-468. Doi: 10.1590/S0104-11692012000300006

3 Leddy MA, Power ML, Schulkin J. The impact of maternal obesity on maternal and fetal health. Rev Obstet Gynecol 2008;1(04):170-178

4 Abu-Saad K, Fraser D. Maternal nutrition and birth outcomes. Epidemiol Rev 2010;32:5-25. Doi: 10.1093/epirev/mxq001

5 Triunfo S, Lanzone A. Impact of maternal under nutrition on obstetric outcomes. J Endocrinol Invest 2015;38(01):31-38. Doi: 10.1007/s40618-014-0168-4

6 Tenenbaum-Gavish K, Hod M. Impact of maternal obesity on fetal health. Fetal Diagn Ther 2013;34(01):1-7. Doi: 10.1159/000350170

7 Chu SY, Kim SY, Lau J, et al. Maternal obesity and risk of stillbirth: a metaanalysis. Am J Obstet Gynecol 2007;197(03):223-228. Doi: 10.1016/j.ajog.2007.03.027

8 Ramachenderan J, Bradford J, McLean M. Maternal obesity and pregnancy complications: a review. Aust N Z J Obstet Gynaecol 2008;48(03):228-235. Doi: 10.1111/j.1479-828X.2008.00860.x

9 Ebrahimi F, ShariffZM, Tabatabaei SZ, Fathollahi MS, Mun CY, Nazari M. Relationship between sociodemographics, dietary intake, and physical activity with gestational weight gain among pregnant women in Rafsanjan City, Iran. J Health Popul Nutr 2015;33(01):168-176

10 World Health Organization. Congenital anomalies. Fact Sheets. September 7, 2016http://www.who.int/mediacentre/factsheets/ fs370/en/. Accessed January 25, 2017

11 Sitkin NA, Farmer DL. Congenital anomalies in the context of global surgery. Semin Pediatr Surg 2016;25(01):15-18. Doi: 10.1053/j.sempedsurg.2015.09.004

12 Correa A, Marcinkevage J. Prepregnancy obesity and the risk of birth defects: an update. Nutr Rev 2013;71(Suppl 1):S68-S77. Doi: $10.1111 /$ nure. 12058

13 Hildebrand E, Gottvall T, Blomberg M. Maternal obesity and detection rate of fetal structural anomalies. Fetal Diagn Ther 2013;33(04):246-251. Doi: 10.1159/000343219

14 Villar J, Merialdi M, Gülmezoglu AM, et al. Nutritional interventions during pregnancy for the prevention or treatment of maternal morbidity and preterm delivery: an overview of randomized controlled trials. J Nutr 2003;133(05, Suppl 2):1606S-1625S. Doi: 10.1093/jn/133.5.1606S

15 Macumber I, Schwartz S, Leca N. Maternal obesity is associated with congenital anomalies of the kidney and urinary tract in offspring. Pediatr Nephrol 2017;32(04):635-642. Doi: 10.1007/ s00467-016-3543-x

16 Blomberg MI, Källén B. Maternal obesity and morbid obesity: the risk for birth defects in the offspring. Birth Defects Res A Clin Mol Teratol 2010;88(01):35-40. Doi: 10.1002/bdra.20620

17 Stothard KJ, Tennant PW, Bell R, Rankin J. Maternal overweight and obesity and the risk of congenital anomalies: a systematic review and meta-analysis. JAMA 2009;301(06):636-650. Doi: 10.1001/jama.2009.113

18 Taruscio D, Arriola L, Baldi F, et al. European recommendations for primary prevention of congenital anomalies: a joined effort of EUROCAT and EUROPLAN projects to facilitate inclusion of this topic in the National Rare Disease Plans. Public Health Genomics 2015;18:184-191. Doi: 10.1159/000360602

19 Atalah E, Castillo C, Castro R, Aldea A. [Proposal of a new standard for the nutritional assessment of pregnant women]. Rev Med Chil 1997;125(12):1429-1436

20 Institute of Medicine. National Research Council. Committee to Reexamine IOM Pregnancy Weight Guidelines. Weight Gain During Pregnancy: Reexamining the Guidelines. Washington, DC: The National Academies Press; 2009https://www.cbsnews.com/htdocs/ pdf/052809_pregnancy.pdf. Accessed January 25, 2017
21 Marwah S, Sharma S, Kaur H, Gupta M, Goraya SPS. Surveillance of congenital malformations and their possible risk factors in a teaching hospital in Punjab. Int J Reprod Contracept Obstet Gynecol 2014;3(01):162-167. Doi: 10.5455/2320-1770.ijrcog20140332

22 Sunitha T, Rebekah K, Muni T, et al. Risk factors for congenital anomalies in high risk pregnant women: a large study from South India. Egypt J Med Hum Genet 2017;18(01):79-85. Doi: 10.1016/ j.ejmhg.2016.04.001

23 Prashar N, Gupta S, Thakur R, Sharma P, Sharma G. A study of incidence of congenital anomalies in newborn: a hospital based study. Int J Res Med Sci. 2016;4(06):2050-2053

24 Li K, Wahlqvist ML, Li D. Nutrition, one-carbon metabolism and neural tube: a review. Nutrients 2016;8(11):E741. Doi: 10.3390/ nu8110741

25 Singh N, Kumble Bhat V, Tiwari A, et al. A homozygous mutation in TRIM36 causes autosomal recessive anencephaly in an Indian family. Hum Mol Genet 2017;26(06):1104-1114. Doi: 10.1093/ $\mathrm{hmg} / \mathrm{ddx} 020$

26 Siega-Riz AM, Viswanathan M, Moos MK, et al. A systematic review of outcomes of maternal weight gain according to the Institute of Medicine recommendations: birthweight, fetal growth, and postpartum weight retention. Am J Obstet Gynecol 2009;201(04):339.e1-339.e14. Doi: 10.1016/ j.ajog.2009.07.002

27 Athukorala C, Rumbold AR, Willson KJ, Crowther CA. The risk of adverse pregnancy outcomes in women who are overweight or obese. BMC Pregnancy Childbirth 2010;10:56. Doi: 10.1186/ 1471-2393-10-56

28 Rankin J, Tennant PW, Stothard KJ, Bythell M, Summerbell CD, Bell R. Maternal body mass index and congenital anomaly risk: a cohort study. Int J Obes 2010;34(09):1371-1380. Doi: 10.1038/ ijo.2010.66

29 Marchi J, Berg M, Dencker A, Olander EK, Begley C. Risks associated with obesity in pregnancy, for the mother and baby: a systematic review of reviews. Obes Rev 2015;16(08):621-638. Doi: $10.1111 /$ obr.12288

30 Sachdeva P, Patel BG, Patel BK. Drug use in pregnancy; a point to ponder!. Indian J Pharm Sci 2009;71(01):1-7. Doi: 10.4103/0250474X.51941

31 Crighton E, Abelsohn A, Blake J, et al. Beyond alcohol and tobacco smoke: Are we doing enough to reduce fetal toxicant exposure? J Obstet Gynaecol Can 2016;38(01):56-59. Doi: 10.1016/j.jogc. 2015.10.009

32 Ortega-García JA, Gutierrez-Churango JE, Sánchez-Sauco MF, et al. Head circumference at birth and exposure to tobacco, alcohol and illegal drugs during early pregnancy. Childs Nerv Syst 2012;28 (03):433-439. Doi: 10.1007/s00381-011-1607-6

33 Calone A, Madi JM, Araújo BF, et al. [Congenital defects: maternal and perinatal features]. Rev AMRIGS. 2009;53:226-230

34 Silva RMM, Mazotti BR, Zilly A, Ferreira H, Caldeira S. [Epidemiologic factors correlated to the risk for fetal death: integrative review]. Arq Ciênc Saúde. 2016;23:9-15. Doi: 10.17696/23183691.23.2.2016.221

35 Noronha Neto C, Souza ASR, Moraes Filho OB, Noronha AMB. [Validation of ultrasound diagnosis of fetal anomalies at a reference center in Pernambuco]. Rev Assoc Med Bras (1992) 2009;55 (05):541-546. Doi: 10.1590/S0104-42302009000500016

36 Rasouly HM, Lu W. Lower urinary tract development and disease. Wiley Interdiscip Rev Syst Biol Med 2013;5(03):307-342. Doi: 10.1002/wsbm.1212

37 Best KE, Tennant PW, Bell R, Rankin J. Impact of maternal body mass index on the antenatal detection of congenital anomalies. BJOG 2012;119(12):1503-1511. Doi: 10.1111/j.1471-0528.2012. 03462.x

38 Dashe JS, McIntire DD, Twickler DM. Maternal obesity limits the ultrasound evaluation of fetal anatomy. J Ultrasound Med 2009; 28(08):1025-1030. Doi: 10.7863/jum.2009.28.8.1025 
39 Zozzaro-Smith P, Gray LM, Bacak SJ, Thornburg LL. Limitations of aneuploidy and anomaly detection in the obese patient. J Clin Med 2014;3(03):795-808. Doi: 10.3390/jcm3030795

40 Persson M, Cnattingius S, Villamor E, et al. Risk of major congenital malformations in relation to maternal overweight and obesity severity: cohort study of 1.2 million singletons. BMJ 2017;357: j2563. Doi: 10.1136/bmj.j2563

41 Longstreet B, Balakrishnan K, Saltzman B, Perkins JA, Dighe M. Prognostic value of a simplified anatomically based nomenclature for fetal nuchal lymphatic anomalies. Otolaryngol Head Neck Surg 2015;152(02):342-347. Doi: 10.1177/0194599814559190

42 Bernal LM, López G. Diagnóstico prénatal: retrospectiva. Nova 2014;12(21):23-36

43 Ebara S. Nutritional role of folate. Congenit Anom (Kyoto) 2017;57 (05):138-141. Doi: 10.1111/cga.12233

44 Mattar R, Torloni MR, Betrán AP, Merialdi M. [Obesity and pregnancy]. Rev Bras Ginecol Obstet 2009;31(03):107-110. Doi: 10.1590/S0100-72032009000300001
45 Zhao Z. TGFß and Wnt in cardiac outflow tract defects in offspring of diabetic pregnancies. Birth Defects Res B Dev Reprod Toxicol 2014;101(05):364-370. Doi: 10.1002/bdrb.21120

46 Saad MI, Abdelkhalek TM, Saleh MM, Haiba MM, Tawfik SH, Kamel MA. Maternal diabetes impairs oxidative and inflammatory response in murine placenta. Springerplus 2016;5:532. Doi: 10.1186/s40064-016-2180-y

47 Gabbe SG, Graves CR. Management of diabetes mellitus complicating pregnancy. Obstet Gynecol 2003;102(04):857-868. Doi: 10.1016/j.obstetgynecol.2003.07.001

48 Liu X, Liu G, Wang P, et al. Prevalence of congenital heart disease and its related risk indicators among 90,796 Chinese infants aged less than 6 months in Tianjin. Int J Epidemiol 2015;44(03): 884-893. Doi: 10.1093/ije/dyv107

49 Persson M, Pasupathy D, Hanson U, Westgren M, Norman M. Prepregnancy body mass index and the risk of adverse outcome in type 1 diabetic pregnancies: a population-based cohort study. BMJ Open 2012;2(01):e000601. Doi: 10.1136/bmjopen-2011-000601 